\title{
Comparison of diffuse-reflectance absorbance and attenuated total reflectance FT-IR for the discrimination of bacteria
}

\author{
Catherine L. Winder* and Royston Goodacre \\ Department of Chemistry, UMIST, PO Box 88, Sackville Street, Manchester, UK \\ M601QD. E-mail: C.Winder@umist.ac.uk
}

Received 1st June 2004, Accepted 31st August 2004

First published as an Advance Article on the web 4th October 2004

\begin{abstract}
A collection of bacteria belonging to the genus Bacillus were analysed by diffuse reflectance absorbance and attenuated total reflectance Fourier transform infrared (FT-IR) spectroscopy in the mid-infrared. The diffuse reflectance absorbance method is a rapid whole organism fingerprinting method, which generates a biochemical profile of the bacteria, where samples are presented to the FT-IR spectrometer dried on a metal carrier. The attenuated total reflectance FT-IR used in conjunction with a diamond attenuated total reflectance (ATR) accessory produces a biochemical profile of the surface chemistry of bacteria directly without the need for drying, and has not previously been used in the discrimination of bacteria. Principal component, discriminant function and hierarchical cluster analysis were performed on the data to discriminate the bacteria. The differentiation of the bacteria to species level was observed in both analyses however, it was concluded that the ATR FT-IR illustrated better sub-species differentiation of the microorganisms. This may imply that the total biochemical profiling infers discrimination to species level whereas strain specific markers are present on the cell surface chemistry.
\end{abstract}

\section{Introduction}

The differentiation of microorganisms using genetic and phenotypic characteristics has been extensively studied to produce a rapid, reproducible and inexpensive identification technique. Ideally the technique would effectively identify sources of infection and differentiate to sub-species level. Traditional biochemical testing based on growth and nutritional properties is time consuming and laborious, thereby hindering the effective treatment of microbial infections. The recent development of molecular techniques to characterise microorganisms provides a rapid solution, however, these techniques are expensive and often require highly specialised operation, which limits their use in routine laboratories. Vibrational spectroscopic techniques (Raman and Fourier transform infrared spectroscopy) generate biochemical 'fingerprints', which when coupled to multivariate statistical analysis are demonstrated to discriminate microorganisms to subspecies levels. ${ }^{1-6}$ Numerous advantages of using this method of discrimination over the traditional microbiological and molecular techniques are documented, and these include speed, minimal sample preparation, automation, and relative lack of expense. In addition, recent optimisation of the techniques for implementation into the clinical environment has reduced the amount of sample required, thereby reducing isolate growth and identification times. 6,7

Various FT-IR techniques are used in our laboratory, the most commonly applied method for routine analysis is a highthroughput (HT FT-IR) diffuse reflectance technique. ${ }^{3,4,8,9}$ In this investigation we compared our high-throughput method to a diamond-accessorised attenuated total reflectance (ATR) FTIR. ATR FT-IR has fewer problems with baseline shifts over the high-throughput method, in addition the samples do not require colonies to be put into suspension and then dehydrated resulting in simpler (direct) sample preparation. ${ }^{10}$ The diamond ATR element used in this study provides excellent chemical inertness to chemistry and abrasion, a wide range of optical transparency and a low coefficient of friction, which reduces substance adherence to the surface.
ATR FT-IR using a zinc selenide crystal has previously been applied to discriminate and differentiate bacteria, generally in antimicrobial profiling studies or to study biofilm formation. For example, the differentiation of strains of E. coli $\mathrm{K} 12$ and six transconjugates exhibiting varying levels of tolerance to $\beta$-lactams antibiotics; ${ }^{10}$ and the differentiation of sensitive and resistant strains of Pseudomonas aeruginosa to the antibiotic imipenem. ${ }^{11}$ ATR spectroscopy was previously compared to FT-IR microspectroscopy and confocal near-IR-Raman microspectroscopy to differentiate a collection of Enterococ$c i,{ }^{12}$ and the authors concluded that the classification scheme generated by each method was more-or-less equivalent. ATR spectroscopy has been described as an excellent technique to investigate biofilm formation because it may be used to study the chemical composition of smooth surfaces without disrupting the biofilm substratum/liquid interface. ${ }^{13,14}$ Therefore microbial communities may be investigated in real time studies without their destruction.

To date only $\mathrm{ZnSe}$ crystals have been used in bacterial differentiation studies. ZnSe crystals are not used for routine analysis because they are easily damaged and toxic to the organisms in contact with it, which may affect the biochemical nature of the sample. The only reported study utilising diamond ATR for microbiological applications is in the detection of Fusarium fungi on maize. ${ }^{15}$

This aim of this study was to investigate the discriminative potential of ATR FT-IR spectroscopy using a diamond crystal with single reflection in comparison to the routinely used highthroughput diffuse reflectance FT-IR technique, with a collection of Bacillus subtilis and Bacillus cereus strains.

\section{Materials and methods}

\section{Cultivation and harvesting of bacteria}

Seven strains of B. subtilis and three strains of B. cereus (Table 1) were cultured on blood agar base media at $37{ }^{\circ} \mathrm{C}$ for $18 \mathrm{~h}$. Three individual cultures of each organism (the so-called 'biological' replicates) for both HT FT-IR and ATR FT-IR 
Table 1 Details of the bacteria used in the investigation

\begin{tabular}{lrll}
\hline Species & Reference & LMG no. & Source \\
\hline Bacillus subtilis & S10 & $7135^{\mathrm{T}}$ & DSM 28 \\
Bacillus subtilis & $\mathrm{S} 12$ & 12260 & Lactose fermenter, Norris \\
Bacillus subtilis & $\mathrm{S} 8$ & 12264 & Gibson \\
Bacillus subtilis & $\mathrm{S} 9$ & & Gibson \\
Bacillus subtilis & $\mathrm{S} 11$ & 17721 & Curry, Colindale \\
Bacillus subtilis & $\mathrm{S} 7$ & 17722 & Cocoa, Carr \\
Bacillus subtilis & $\mathrm{S} 6$ & & Spore strip, Halls \\
Bacillus cereus & $\mathrm{C} 25$ & 17608 & Gordon \\
Bacillus cereus & $\mathrm{C} 26$ & 17619 & Mastitis, Fishlock \\
Bacillus cereus & $\mathrm{C} 27$ & 17610 & Blood, Gordon \\
\hline
\end{tabular}

were analysed: three replicates of each biological replicate were collected (the so-called 'machine' replicates).

\section{Diffuse reflectance-absorbance HT FT-IR spectroscopy}

The cells were harvested from agar plates into $800 \mu \mathrm{l}$ physiological saline $(0.9 \% \mathrm{NaCl})$ and stored on ice until required. A $10 \times 10 \mathrm{~cm}$ aluminium plate was rinsed with acetone and dried at $50{ }^{\circ} \mathrm{C}$ for $10 \mathrm{~min}$. FT-IR analysis was initially performed on the plate without samples to provide a reference reading for each well. The plate was loaded onto the motorised stage of a reflectance TLC accessory, ${ }^{2,16-18}$ attached to a Bruker IFS28 FT-IR spectrometer (Bruker Spectrospin Ltd., Coventry, UK). This was equipped with a mercury-cadmiumtelluride (MCT) detector, which was cooled with liquid $\mathrm{N}_{2}$. A $10 \mu \mathrm{l}$ aliquot of each sample was evenly applied in triplicate onto the 100 well plate (the machine replicates). The plate was then dried at $50{ }^{\circ} \mathrm{C}$ for $30 \mathrm{~min}$ before FT-IR analysis was performed.

\section{Diamond ATR FT-IR spectroscopy of the bacteria}

FT-IR was performed using a diamond ATR accessory (DuraSamplIR $\mathrm{IM}$, SensIR Technologies, Danbury, kindly on loan from Alan Sanders) for FT-IR spectrometers on an IFS28 infrared spectrometer equipped with a deuterated triglycine sulfate (DTGS) detector. The diamond ATR sensor has a single-reflection Duradisk (IM) suitable for high-throughput applications. The diamond has a $0.5 \mathrm{~mm}$ thickness and $1 \mathrm{~mm}$ diameter with a $200 \mu \mathrm{m}$ 'sweet spot', it is mounted flush with the plate for optimal sample contact. The diamond disc is interfaced to a $\mathrm{ZnSe}$ focussing element which provides the interfacing optics for the input and output radiation.

A colony of bacteria was carefully removed from the agar plate using a plastic loop, taking care not to disrupt the agar. The biomass was pressed against the diamond crystal of the ATR device with a pressure applicator. The torque knob on the pressure applicator ensured that the same pressure was applied to all the samples. A background measurement of the surrounding environment was taken before each sample was applied to the crystal. The crystal was cleaned with $70 \%$ ethanol and then acetone between samples.

\section{Spectral acquisition}

Spectra were collected over the wavelength range of 4000 to $600 \mathrm{~cm}^{-1}$ (HT FT-IR) and 4000 to $900 \mathrm{~cm}^{-1}$ (ATR FT-IR; a reduced range was used because of the $\mathrm{ZnSe}$ focussing element) under the control of an IBM-compatible computer programmed with OPUS 2.1 running under IBM OS/2 Warp, which was provided by the manufacturers. Spectra were acquired with a resolution of $4 \mathrm{~cm}^{-1}$, and 256 spectra were co-added and averaged to improve the signal to noise ratio. The collection time for the spectra was approximately $10 \mathrm{~s}$ per sample for the HT FT-IR (MCT detector employed) and $60 \mathrm{~s}$ for the ATR FT-IR (DTGS detector used). The spectra are displayed in terms of absorbance, which was calculated from the reflectance-absorbance spectra using OPUS software. Typical unprocessed spectra are shown in Fig. 1.

\section{Preprocessing}

The ASCII data were imported into Matlab version 6 (The MathWorks, Inc., MA, USA). To minimize problems arising from baseline shifts Matlab was used to correct for $\mathrm{CO}_{2}$ vibrations (the $\mathrm{CO}_{2}$ peaks at $2403-2272 \mathrm{~cm}^{-1}$ and 683 $656 \mathrm{~cm}^{-1}$ were removed, and filled with a trend) and windows of the spectra likely containing $\mathrm{H}_{2} \mathrm{O}$ vibrations were smoothed with a window of $35 \mathrm{~cm}^{-1}$ to reduce noise (for TLC-FTIR this was 4000-3036 $\mathrm{cm}^{-1}$ and 2458-1706 $\mathrm{cm}^{-1}$; whilst for ATRFTIR this was $2725-1556 \mathrm{~cm}^{-1}$ ). The spectra were normalised such that the smallest recorded absorbance was set to 0 and the highest was set to 1 for each spectrum and then the first derivatives (Savitzky \& Golay with a window of 5) were used for cluster analysis.

\section{Cluster analyses}

Principal components analysis $\left(\mathrm{PCA}^{19}\right)$ was performed on the spectra to reduce the dimensionality of the multivariate data whilst preserving the variance, prior to discriminant function analysis (DFA). DFA is a supervised technique which discriminates between groups on the basis of the retained principal components with a priori knowledge of which spectra were replicates. ${ }^{20,21}$ The DFA minimises 'within group' variance and maximises 'between group' variance. The data

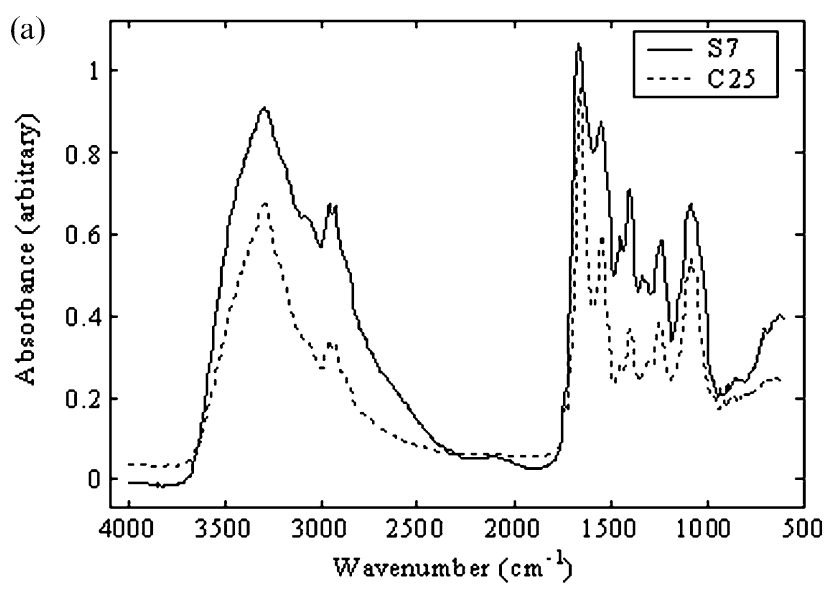

(b)

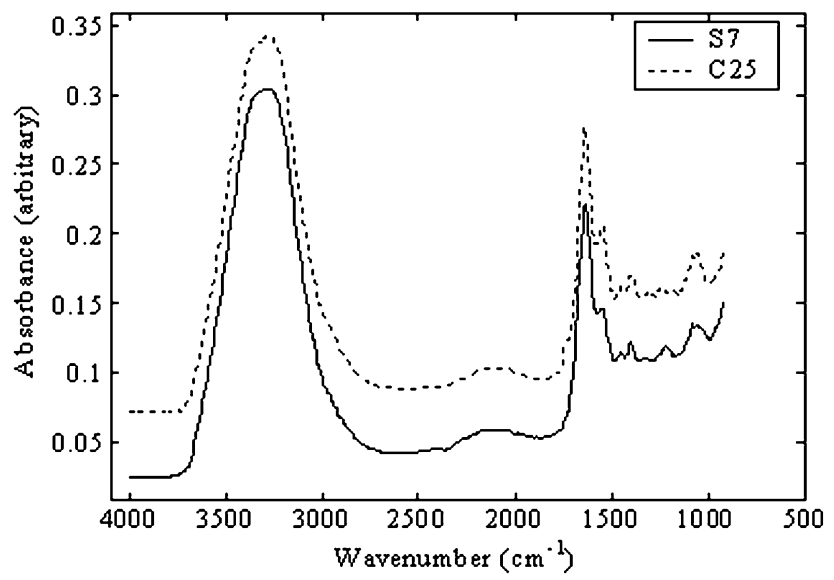

Fig. 1 Examples of typical spectra analysed with the high throughput FT-IR (a) and ATR FT-IR (b). Bacillus subtilis (S7) and Bacillus cereus (C25) are shown, the baseline of $\mathrm{C} 25$ is offset so that both spectra can be seen more clearly. 
set was validated to ensure the correct number of principal components (PCs) and discriminant functions (DFs) were selected. In this process PCA and DFA were performed on a subset of the data using 2 a priori classes per strain (i.e., at the level of the machine replicates and so this does not bias the analysis), the 'training data' (replicates 1 to 6 , samples designated $\mathrm{a}$ and $\mathrm{b}$ for the first two biological replicates). The remaining replicates, the 'test data' (replicates 7 to 9, samples designated c) were projected into the $\mathrm{PC}$ and $\mathrm{DF}$ space to ensure that the replicates from the test data cluster with those from the training data. Finally, hierarchical cluster analysis (HCA) was used to construct a dendrogram from the a priori group centres in the DFA space, using scaled Euclidean distances as described by Goodacre et al., ${ }^{3}$ and the dendrogram was produced using average linkage clustering algorithm. ${ }^{22}$

\section{Results and discussion}

\section{HT FT-IR spectroscopy}

Examples of typical raw spectra of the bacilli collected with the high throughput technique are shown in Fig. 1a. Minimal qualitative differences are observed between the two samples (B. subtilis, $\mathrm{S} 7$ and $B$. cereus, $\mathrm{C} 25$ ), however considerable quantitative differences are clearly visible. The baseline of the second sample (C25) is slightly offset in the figure to illustrate the contours of each sample. Due to the complex nature of the data, multivariate data analysis was used to discriminate the species and strains of bacilli.

PCA of the first derivative spectra indicated species discrimination (data not shown) of the bacilli samples and strain discrimination of the three strains of B. cereus. However, the PCA failed to resolve the B. subtilis samples to the strain level, hence DFA was performed on the retained principal components with the a priori knowledge of the biological replicates (replicates ' $a$ ' and ' $b$ ' in the plots). The analysis was validated on the basis of the third biological replicate (marked ' $c$ ' in the plots), the DFA was performed on the training data ('a' and 'b') and the test data ('c') was projected into the discriminant function (DF) space. A distinction between the two species of bacilli was initially observed from the DFA plot (Fig. 2a, DF1 vs. DF2). Three independent clusters corresponding to the three strains of $B$. cereus were observed within the cluster of $B$. cereus replicates (Fig. 2a) and in the branching pattern from the HCA (Fig. 2b). The seven strains of B. subtilis analysed were recovered in three clusters (Fig. 2a). The dendrogram (Fig. 2b) illustrates the clustering of the B. subtilis strains, the type strain $\mathrm{S} 10$ clusters with the two strains (S7 and S11) isolated from foodstuffs (Table 1), S8 was recovered with $\mathrm{S} 12$, and $\mathrm{S} 6$ clusters with $\mathrm{S} 9$. In general the sources of isolation (Table 1) do not suggest any reason to explain the clustering of the B. subtilis isolates.

The differentiation of the strains of $B$. subtilis improved slightly when re-analysed separately from the B. cereus strains (Fig. 3). The replicates of S7 clustered separately from those of S10 and S11 and the replicates of S6 were discriminated from those of S9. However, the replicates of S8 and S12 are still recovered together.

\section{ATR FT-IR spectroscopy}

Typical examples of raw spectra taken with the diamond ATR FT-IR spectroscopy are shown in Fig. 1b. As previously observed with the HT FT-IR technique few qualitative differences were observed between the two examples shown (B. subtilis, $\mathrm{S} 7$ and B. cereus, $\mathrm{C} 25$ ), however, quantitative differences are visible particularly in the spectral region of 1200 to $1600 \mathrm{~cm}^{-1}$. PCA (data not shown) of the data did not illustrate discrimination of the bacteria and the analysis was not improved by further spectral processing (e.g., different

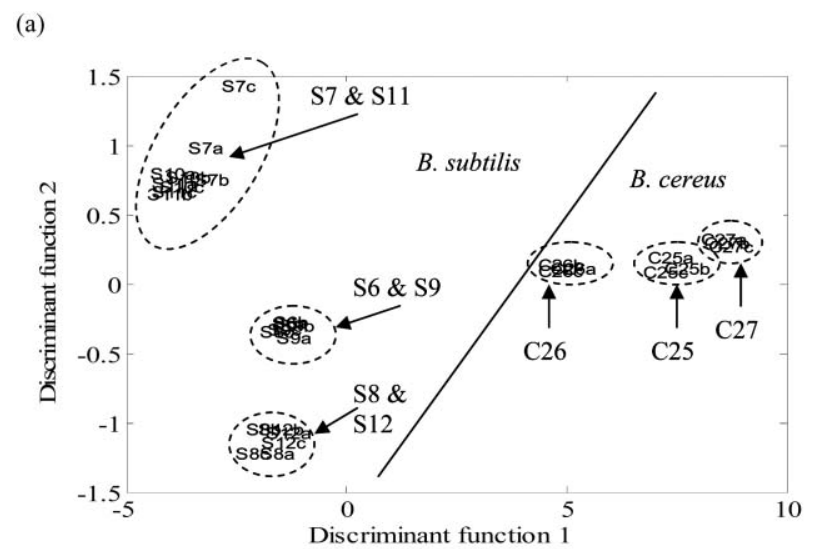

(b)

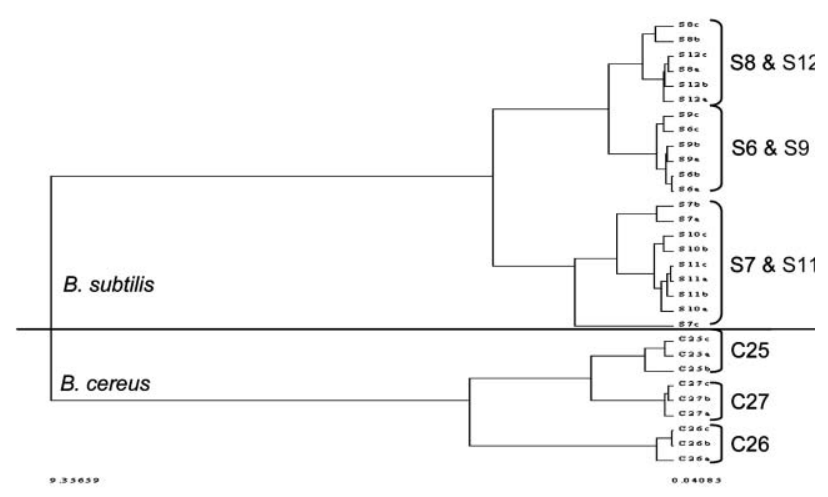

Fig. 2 Clustering of the data collected with the high-throughput FTIR by (a) DFA and (b) HCA. PCs 1-9 (total cumulative variance is $95.34 \%$ ) were used by the DFA algorithm with a priori knowledge of machine replicates and DFs 1-3 used for HCA. Refer to Table 1 for strain designation details.

derivatisation windows and taking the 2nd derivative). However, using the supervised technique DFA (Fig. 4a; showing the a priori group centres) the bacteria were clearly separated according to species. The biological replicates of five out of the seven strains of $B$. subtilis were recovered to their strain levels from the analysis (Fig. 4a; DF 1 vs. DF 2). The HCA (Fig. 4b) illustrates the recovery of all seven strains of B. subtilis. The B. cereus strains are not clearly differentiated from the DFA (Fig. 4a) and although the three replicates of strain $\mathrm{C} 27$ are recovered together from the HCA (Fig. 4b), the remaining two strains of $B$. cereus are not adequately discriminated by the analysis.

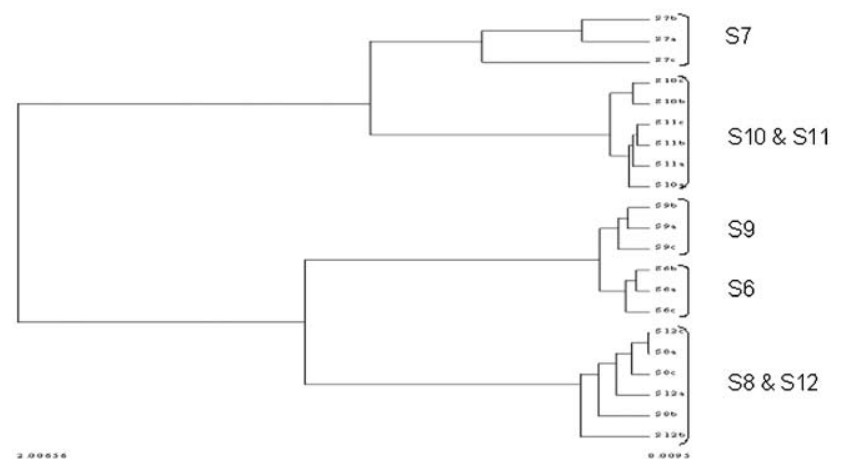

Fig. 3 Dendrogram of the subgroup analysis for the B. subtilis strains by high-throughput FT-IR (PCs 1-5 followed by DFs 1-3). Refer to Table 1 for strain designation details. 


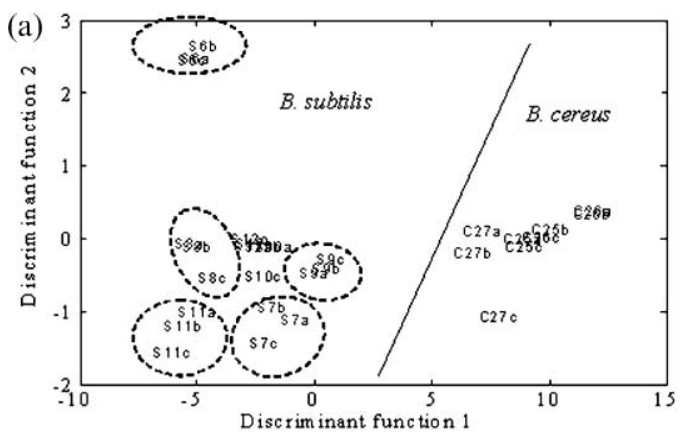

(b)

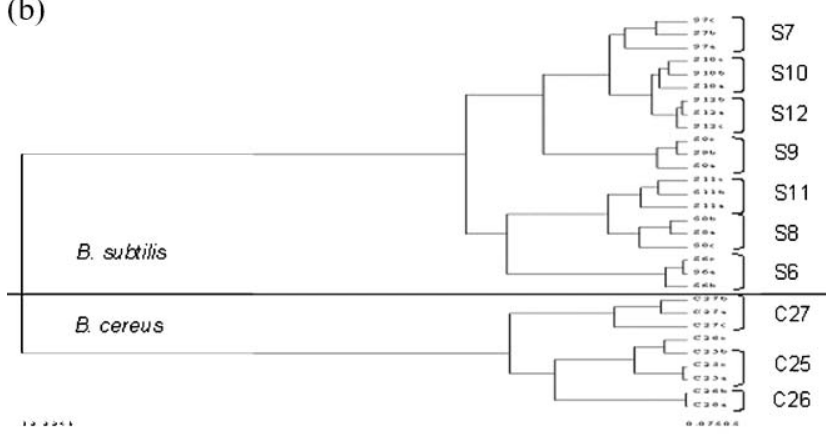

Fig. 4 Clustering of the data collected with the ATR FT-IR by (a) DFA and (b) HCA. Ellipses in DFA are drawn as a guide and have no statistical significance and are referred to in the text. PCs 1-20 (total cumulative variance is $99.94 \%$ ) were used by the DFA algorithm with $a$ priori knowledge of machine replicates and DFs $1-4$ were used for HCA. Refer to Table 1 for strain designation details.

\section{Comparison of the two techniques}

The spectra collected with the HT FT-IR contain five spectral windows which are considered important for bacterial discrimination (Table 2). ${ }^{23}$ The most noticeable difference between the HT FT-IR and ATR FT-IR spectra is the absence of $\mathrm{C}-\mathrm{H}$ vibrations leading to peaks in the fatty acid region (W1, Table 2; Fig. 1a). This may be due to the nature of the sample technique, ATR FT-IR spectroscopy detects the surface chemistry of the cells, Bacillus spp. are Gram-positive bacteria and therefore do not contain a high proportion of lipids in the outer cell wall. Note, spectra of mycolic acid containing mycobacteria collected in this laboratory do clearly show $\mathrm{C}-\mathrm{H}$ lipid stretches (data not shown), whereas, the HT FT-IR will penetrate the bacterial cell structure and therefore produce a fingerprint characteristic of the total cell components rather than the outer surface. The protein peaks observed in the region W2 (Table 2) were observed with both collection methods, however, the peak shape of the spectra between 1500 to $900 \mathrm{~cm}^{-1}$ collected with the ATR technique differs greatly from the spectra observed with the HT FT-IR spectroscopy. The 'true' fingerprint region (W5, below $900 \mathrm{~cm}^{-1}$ ) is not present in the spectra collected with the ATR FT-IR spectroscopy, this is because the vibrations of the $\mathrm{ZnSe}$ crystal

Table 2 Important spectral windows for the discrimination of bacteria $^{23}$

\begin{tabular}{|c|c|c|}
\hline $\begin{array}{l}\text { Spectral } \\
\text { window }\end{array}$ & $\begin{array}{l}\text { Wavenumber } \\
\text { range/ } / \mathrm{cm}^{-1}\end{array}$ & Dominant compounds \\
\hline W1 & $3000-2800$ & Fatty acids \\
\hline W2 & $1700-1500$ & Proteins \\
\hline \multirow[t]{2}{*}{ W3 } & $1450-1200$ & $\begin{array}{l}\text { Carboxylic groups of proteins, } \\
\text { free amino acids, polysaccharides }\end{array}$ \\
\hline & $(1250-1200)$ & RNA/DNA, phospholipids \\
\hline W4 & $1200-900$ & Polysaccharides \\
\hline W5 & $<900$ & True fingerprint region \\
\hline
\end{tabular}

are detected in this region, which will mask any vibrations originating from the sample.

Species discrimination of the samples was observed with both techniques (Figs. 2 and 4). However, the strain differentiation of the bacilli was better with the data collected from ATR FT-IR spectroscopy. The analysis of the data collected by the HT FT-IR illustrated differentiation of the three strains of $B$. cereus, however, the seven strains of $B$. subtilis were recovered in three clusters (Fig. 2b). The subgroup analysis of only the strains of $B$. subtilis slightly improved the discrimination of these bacilli (Fig. 3a), however, there was still some inter-mixing of the strains. Whilst for the ATR FT-IR data some intermixing of the strains of $B$. cereus was observed (Fig. 4), clear differentiation of the B. subtilis strains was seen. The fingerprint acquired with ATR FT-IR technique is considered to be characteristic of the cell surface chemistry rather than the entire cell biochemistry (HT FT-IR), this therefore implies the surface chemistry of the cell is sufficient to differentiate these bacilli cells to sub-species level.

\section{Conclusions}

The discrimination of these bacteria to species level was observed with both FT-IR techniques. However, in this comparative analysis the ATR FT-IR technique illustrated better strain differentiation of the Bacillus subtilis. This may imply strain specific markers are present on the cell wall surface, whereas, the total biochemical cell components predominantly infer discrimination according to species. The results observed in this study are preliminary, in future studies the number of species and strains will be increased to investigate the discriminatory potential of the ATR FT-IR technique further.

\section{Acknowledgements}

We would like to thank Alan Sanders for kindly loaning the diamond ATR accessory, Dave Ellis for his guidance and assistance in the operation of the FT-IR spectroscopy and Niall Logan for supplying the bacteria.

\section{References}

1 D. Naumann, D. Helm and H. Labischinski, Nature, 1991, 351 81-82.

2 R. Goodacre, E. M. Timmins, P. J. Rooney, J. J. Rowland and D. B. Kell, FEMS Microbiol. Lett., 1996, 140, 233-239.

3 R. Goodacre, E. M. Timmins, R. Burton, N. Kaderbhai, A. M. Woodward, D. B. Kell and P. J. Rooney, Microbiology (UK), 1998, 144, 1157-1170

4 E. M. Timmins, S. A. Howell, B. K. Alsberg, W. C. Noble and R. Goodacre, J. Clin. Microbiol., 1998, 36, 367-374.

5 K. Maquelin, C. Kirschner, L. P. Choo-Smith, N. van den Braak, H. P. Endtz, D. Naumann and G. J. Puppels, J. Microbiol. Methods, 2002, 51, 255-271.

6 K. Maquelin, C. Kirschner, L. P. Choo-Smith, N. A. Ngo-Thi, T. van Vreeswijk, M. Stammler, H. P. Endtz, H. A. Bruining, D. Naumann and G. J. Puppels, J. Clin. Microbiol., 2003, 41, 324-329.

7 K. Maquelin, L. P. Choo-Smith, T. van Vreeswijk, H. P. Endtz, B. Smith, R. Bennett, H. A. Bruining and G. J. Puppels, Anal. Chem., 2000, 72, 12-19.

8 C. L. Winder, E. Carr, R. Goodacre and R. Seviour, J. Appl. Microbiol., 2004, 96, 328-339.

9 S. Lai, R. Goodacre and L. N. Manchester, Syst. Appl. Microbiol., 2004, 27, 186-191.

10 W. Bouhedja, G. D. Sockalingum, P. Pina, P. Allouch, C. Bloy, R. Labia, J. M. Millot and M. Manfait, FEBS Lett., 1997, 412, $39-42$.

11 G. D. Sockalingum, W. Bouhedja, P. Pina, P. Allouch, C. Mandray, R. Labia, J. M. Millot and M. Manfait, Biochem. Biophys. Res. Commun., 1997, 232, 240-246. 
12 C. Kirschner, K. Maquelin, P. Pina, N. A. N. Thi, L. P. ChooSmith, G. D. Sockalingum, C. Sandt, D. Ami, F. Orsini, S. M. Doglia, P. Allouch, M. Mainfait, G. J. Puppels and D. Naumann, J. Clin. Microbiol., 2001, 39, 1763-1770.

13 J. Schmitt and H. C. Flemming, Int. Biodeterior. Biodegrad., 1998, 41, 1-11.

14 P. A. Suci, J. D. Vrany and M. W. Mittelman, Biomaterials, 1998, 19, 327-339.

15 G. Kos, H. Lohninger and R. Krska, Vib. Spectrosc., 2002, 29, 115-119.

16 G. Glauninger, K. A. Kovar and V. Hoffmann, Fresenius' J. Anal. Chem., 1990, 338, 710-716.

17 S. P. Bouffard, J. E. Katon, A. J. Sommer and N. D. Danielson, Anal. Chem., 1994, 66, 1937-1940.
18 M. K. Winson, R. Goodacre, E. M. Timmins, A. Jones, B. K. Alsberg, A. M. Woodward, J. J. Rowland and D. B. Kell, Anal. Chim. Acta, 1997, 348, 273-282.

19 I. Jolliffe, Principal Component Analysis, Springer-Verlag, New York, 1986

20 H. J. H. MacFie, C. S. Gutteridge and J. R. Norris, J. Gen. Microbiol., 1978, 104, 67-74.

21 W. Windig, J. Haverkamp and P. G. Kistemaker, Anal. Chem., 1983, 55, 81-88.

22 B. F. J. Manly, Multivariate Statistical Methods: A Primer, Chapman \& Hall, London, 1994.

23 T. Udelhoven, D. Naumann and J. Schmitt, Appl. Spectrosc., 2000, 54, 1471-1479. 